\title{
Salud vascular y pandemia: percepción del paciente en la consulta vascular ambulatoria durante COVID-19
}

\section{Vascular health and pandemic: Patients perception of vascular ambulatory care during COVID-19}

\author{
Federico López-Uribe ${ }^{1 *}$, Hernán C. Luna ${ }^{1}$, Laura Ma. Contreras-Correa ${ }^{1}$, Martha O. Correa-Posada ${ }^{1,2}$ y \\ John F. García-Vélez ${ }^{2}$
}

${ }^{1}$ Facultad de Medicina, Departamento de Cirugía General, Universidad CES; ${ }^{2}$ Facultad de Medicina, Departamento de Cirugía Vascular, Universidad de Antioquia. Antioquia, Colombia

\begin{abstract}
Resumen
Antecedentes: El COVID-19 desplazó la atención de las enfermedades no urgentes, aumentando la percepción del deterioro en salud, las quejas por demoras en la atención e insatisfacción con la consulta virtual. Objetivo: Determinar la percepción del paciente en la salud vascular y el uso de telemedicina durante la pandemia. Material y métodos: Encuesta en 142 pacientes de consulta de cirugía vascular entre abril y junio de 2021. Resultados: La mayoría tenía enfermedad venosa (EV) seguida de arterial (EA) y linfática (EL). Los pacientes con EA empeoraron los síntomas, mientras que los de EV o EL permanecieron igual. Menos de la mitad tuvo miedo a la atención médica presencial o la suspendieron voluntariamente por temor al contagio de COVID-19. Hubo reprogramación oportuna y satisfacción con la consulta virtual en la mayoría, sin repercusión en la percepción final del estado de salud vascular. Conclusión: Se encontró un aumento de síntomas y disminución en la oportunidad de atención, sin impacto significativo en la salud vascular o complicaciones.
\end{abstract}

Palabras clave: Enfermedades vasculares. Infecciones por coronavirus. COVID-19. Pandemias. Atención ambulatoria.

\begin{abstract}
Background: COVID-19 shifted the attention of the non-urgent disease, increasing the perception of deterioration in health, complaints for delays in patient care and dissatisfaction of the virtual consultation. Objective: To determine the patient's perception of the vascular health deterioration, and the fact of being subjected to telemedicine. Material and methods: Survey on 142 outpatient vascular surgery consultants between April and June 2021. Results: The majority had venous diseases (VD), the others were arterial diseases (AD and lymphatic diseases (LD). AD patients worsened symptoms during the pandemic; most of VD and LD patients remained the same; less than half of them were afraid of seeking in-person attention because of a possible COVID-19 infection, but they were rescheduled in a timely manner and were satisfied with the virtual consultation without changes in the overall perception of their vascular health. Conclusion: An increase in symptoms and a decrease on the opportunity for care were found, there was no significant impact on vascular health or complications.
\end{abstract}

Key words: Vascular diseases. Coronavirus infections. COVID-19. Pandemics. Ambulatory care.

\section{Correspondencia:}

${ }^{*}$ Federico López-Uribe

E-mail: fedelourib@gmail.com
Fecha de recepción: 01-09-2021

Fecha de aceptación: 28-09-2021

DOI: 10.24875/RMA.21000039
Disponible en internet: 29-11-2021

Rev Mex Angiol. 2021;49(4):107-112

www.RMAngiologia.com bajo la licencia CC BY-NC-ND license (http://creativecommons.org/licenses/by-nc-nd/4.0/). 


\section{Introducción}

En la época de la pandemia la atención en muchas especialidades, incluyendo la cirugía vascular, se ha visto enfrentada a cambios en su rutina, tanto para el médico como para el paciente. Los sistemas de salud de todo el mundo han tenido que hacer cambios sustanciales en sus modelos de atención, acelerando el desarrollo de las tecnologías de las comunicaciones como herramienta fundamental en la atención y seguimiento de los pacientes, sin embargo, esto no está al alcance de todas las personas. Además, el alto flujo de pacientes con enfermedad por coronavirus 2019 (COVID-19) congestionó los servicios de urgencias y las salas de cuidados intensivos, con lo que la atención de las patologías no urgentes se vio detenida en múltiples escenarios ${ }^{1,2}$. Esto puede causar un gran impacto en la situación de salud de las personas y un detrimento en la calidad de vida. El manejo de la patología urgente de origen vascular posiblemente no se ha visto tan afectado, sin embargo en lugares con recursos limitados sí puede estarlo, y por su carácter urgente puede ser sujeto a mayor control o visualización, pero es más difícil conocer el impacto en la patología no urgente como el manejo de enfermedad arterial (EA) en claudicantes o la enfermedad venosa (EV). El objetivo del estudio fue conocer la percepción de los pacientes con relación con su enfermedad vascular durante la pandemia, aumento de síntomas, aparición de complicaciones, el retraso en la atención y el uso de telemedicina para resolver los problemas no urgentes.

\section{Materiales y métodos}

Se realizó un estudio descriptivo, prospectivo, de corte transversal, por medio de una encuesta de 15 preguntas (con respuestas sí - no) a pacientes que ingresan a la consulta presencial por patología vascular en un centro de enfermedades vasculares en la ciudad de Medellín, (Colombia), en el periodo del 1 de abril de 2021 hasta el 30 de junio de 2021. El cuestionario fue diseñado por los investigadores, con base en las quejas más frecuentes encontradas en la consulta; no ha sido validado, sin embargo, no existe a la fecha ningún cuestionario al respecto validado en la literatura. Las preguntas se hicieron con respecto al último año, e incluyeron cuatro bloques principales: el primero fueron datos demográficos como edad, escolaridad y lugar de residencia; un segundo bloque sobre la asistencia a consultas o la cancelación de procedimientos por parte del sistema o temor del paciente durante el año anterior; un tercer bloque sobre síntomas y complicaciones en el último año, y un cuarto sobre atención virtual con telemedicina en cualquier forma en el mismo periodo. Inicialmente se realizó una prueba piloto con cinco encuestas para evaluar la facilidad de entendimiento de las preguntas y se corrigieron los posibles errores en la elaboración del cuestionario. La encuesta se realizaba antes del ingreso a la consulta, de forma voluntaria y anónima, y se anotaba el diagnóstico al final de la evaluación. No se tuvo en cuenta el aseguramiento del paciente, aunque la mayoría de los atendidos tienen cobertura por el régimen de seguridad social colombiano. Se excluyeron las encuestas que no fueran llenadas completamente o mal diligenciadas, así como los pacientes que no pudieran Ilenarla por sí solos. Las variables cualitativas se expresaron en forma de porcentajes, el análisis estadístico se realizó con el programa estadístico SPSS (versión 21.0, SPSS Inc., Chicago, Illinois).

\section{Resultados}

Se realizaron un total de 142 encuestas. El promedio de edad de los encuestados fue de 55.55 años, con un rango entre 23 y 88 años. La mayoría residían en zona urbana y muy pocos tenían educación superior. La distribución de las variables demográficas recolectadas y de los principales diagnósticos vasculares motivos de consulta se pueden encontrar en la tabla 1.

Los diagnósticos se agruparon según el sistema afectado en causas de EA, EV, enfermedades linfáticas (EL) y otras que incluían diagnósticos mixtos como opérculo torácico, fístulas arteriovenosas y malformaciones. La distribución de los diagnósticos por grupos y la mejoría de la salud vascular global, así como la solución de su problema vascular durante la pandemia puede consultarse en la tabla 2.

El $31 \%$ (44 pacientes) de los encuestados sintió miedo de buscar atención médica presencial y el $15.5 \%$ (22 pacientes) del total de los encuestados suspendieron voluntariamente la atención en salud por temor a ser contagiados de COVID-19.

La suspensión de procedimientos vasculares electivos o citas por vascular por parte de la red de salud a causa de la pandemia se presentó en 23 pacientes (16.2\%), de estos $16(70 \%)$ tuvieron reprogramación, pero solo 9 personas percibieron esta como oportuna.

Más de la mitad de los pacientes $(58 \%, \mathrm{n}=82)$ reportaron aparición de síntomas nuevos relacionados 
Tabla 1. Características demográficas y distribución por diagnósticos de los pacientes encuestados

\begin{tabular}{|l|c|}
\hline Característica & $\mathbf{n}(\%)$ \\
\hline $\begin{array}{l}\text { Residencia } \\
\text { Urbano } \\
\text { Rural }\end{array}$ & $123(86.6 \%)$ \\
\hline $\begin{array}{l}\text { Escolaridad } \\
\text { Básica primaria }\end{array}$ & $19(13.4 \%)$ \\
\hline $\begin{array}{l}\text { Básica secundaria } \\
\text { Superior (técnica, universitaria, posgrados, } \\
\text { maestrías, doctorados) }\end{array}$ & $45(31.7 \%)$ \\
\hline $\begin{array}{l}\text { Enfermedad arterial } \\
\text { Enfermedad arterial oclusiva crónica }\end{array}$ & $53(37.3 \%)$ \\
\hline Aneurismas & $44 \%)$ \\
\hline Enfermedad venosa & \\
\hline Várices & $17(12 \%)$ \\
\hline Úcera venosa & $14(9.9 \%)$ \\
\hline Enfermedad tromboembólica venosa & $3(2.1 \%)$ \\
\hline Enfermedad linfática & $103(72.5 \%)$ \\
\hline Otros & $90(63.4 \%)$ \\
\hline & $7(4.9 \%)$ \\
\hline
\end{tabular}

con su problema vascular en el tiempo evaluado, el $36 \%(n=51)$ refirieron complicaciones nuevas, de las cuales solo 14 las asociaron a demoras en la atención.

La mayoría de los encuestados tenían acceso a los medios para la realización de una consulta $(79.6 \%)$ virtual, siendo mayor en los pacientes a medida que aumenta el grado de escolaridad $(64.4 \%$ en básica primaria, $79 \%$ en secundaria, $96-100 \%$ para educación superior).

Veintiún (14.8\%) pacientes tuvieron cita virtual con el especialista en cirugía vascular, fuera telefónica o por videollamada, de estos, 15 pacientes $(71.4 \%)$ estuvieron satisfechos con la atención y a $14(66.6 \%)$ se les logró solucionar su requerimiento durante esta consulta.

Al preguntar por si han tenido angustia o preocupación por su estado de salud no relacionado con la COVID-19, el 56.3\% (80 pacientes) respondieron que sí. La percepción del estado de salud vascular en general en los pacientes encuestados durante el periodo evaluado fue que la mayoría no sentían ningún cambio ( $n=86,60 \%)$, 31 pacientes se sentían peor $(21.8 \%)$ y $25(17.6 \%)$ referían mejoría de su salud vascular.

\section{Discusión}

Con la llegada de la pandemia muchos hospitales de alta complejidad tuvieron que migrar a ser centros especializados en la atención de pacientes COVID-19 y los médicos de diferentes especialidades, como anestesiología, por ejemplo, tuvieron que cubrir la carencia de recurso humano en áreas como unidades de cuidado intensivo y urgencias para la atención de los pacientes infectados ${ }^{2}$. Esto a su vez llevó a la adopción de políticas de suspender la consulta presencial y la realización de procedimientos electivos tanto diagnósticos como terapéuticos, como ocurrió en Colombia, por un periodo ininterrumpido de casi un año de alerta roja hospitalaria, e hizo que la atención por consulta no prioritaria por cirugía vascular quedara aplazada. Algunas de estas decisiones se basaron en el apoyo de clasificaciones adaptadas que permitían determinar la prioridad según los diagnósticos 3,4 .

Diferentes países han desarrollado guías de atención según sus capacidades, pero el común de países ha diferido los procedimientos y la consulta ambulatoria, como por ejemplo en enfermedades vasculares que no impliquen riesgo inminente para la vida o salud del paciente ${ }^{5}$. El American College of Surgery también propone una clasificación inicial para los pacientes con patología vascular que ayuda a guiar la práctica de los diferentes procedimientos ${ }^{6}$.

Los pacientes encuestados en este trabajo están todos bajo la modalidad de atención ambulatoria en un centro que no realiza urgencias, por esta razón, la mayoría de los pacientes pueden tener un curso más benigno de su enfermedad y no mostrar complicaciones asociadas o ser más tolerantes al retraso de la consulta y de los procedimientos. Las trombosis evaluadas ya habían recibido el manejo en el ámbito agudo y la mayoría de los diagnósticos arteriales como aneurismas fueron atendidos para control o seguimiento de su patología, pero ninguno tuvo que ser referido a urgencias.

Es importante precisar que si bien la reprogramación en esta serie de pacientes no fue percibida como oportuna, el impacto en cuanto a la proporción de complicaciones por el atraso en la atención no fue significativo en este trabajo.

Llama la atención que aunque la percepción de los investigadores en general es que la atención presencial disminuyó por el temor al contagio en el centro, la mayoría de los pacientes entrevistados referían no sentir miedo a buscar atención médica durante la pandemia por COVID-19. No se puede precisar por qué este resultado, es posible que tenga relación con el tiempo en el que se realizó la encuesta, que aunque la región se encontraba en un pico de contagio, ya se había iniciado el desmonte del aislamiento y esto pudo bajar 
Tabla 2. Percepción de la evolución de los síntomas y de la resolución de su problema vascular en los pacientes encuestados

\begin{tabular}{|l|c|c|c|c|c|}
\hline Grupo de diagnóstico & \multicolumn{3}{|c|}{$\begin{array}{c}\text { Percepción global de síntomas } \\
\mathbf{n}(\%)\end{array}$} & \multicolumn{2}{c|}{$\begin{array}{c}\text { Solución de su problema durante la } \\
\text { pandemia } \\
\text { n (\%) }\end{array}$} \\
\cline { 2 - 6 } & Igual & Mejor & Peor & No & Sí \\
\hline Enfermedad arterial & $10(58.8 \%)$ & 0 & $7(41.17 \%)$ & $8(47.5 \%)$ & $9(52.9 \%)$ \\
\hline Enfermedad venosa & $63(61.1 \%)$ & $21(20.4 \%)$ & $19(18.5 \%)$ & $37(35.92 \%)$ & $66(64.07 \%)$ \\
\hline Enfermedad linfática & $6(54.5 \%)$ & $2(18.3 \%)$ & $3(27.2 \%)$ & $4(36.6 \%)$ & $7(63.33 \%)$ \\
\hline Otros diagnósticos & $7(63.3 \%)$ & $2(18.3 \%)$ & $2(18.3 \%)$ & $1(9 \%)$ & $10(91 \%)$ \\
\hline
\end{tabular}

la percepción del riesgo y del temor entre los pacientes.

En EE.UU. se realizó una encuesta a 493 cirujanos donde se encontró que las cirugías electivas fueron las que más se cancelaron, pero los casos de procedimientos para inicio de diálisis, reparaciones aórticas y revascularizaciones de miembros inferiores se han suspendido lo menos posible durante la pandemia por COVID-19. En este estudio la percepción por parte de los cirujanos vasculares de EE.UU. es que hubo una disrupción en el seguimiento clínico ambulatorio de sus pacientes, si bien se tomaron medidas como la teleconsulta hubo pérdidas en el seguimiento. Los procedimientos vasculares se disminuyeron de manera significativa, así como también el uso de los laboratorios vasculares ${ }^{7}$.

Sin embargo, teniendo en cuenta los resultados de este estudio, la percepción del médico puede diferir de la de los pacientes en cuanto a la atención de patología no urgente, ya que, a pesar de la suspensión de la atención y los procedimientos electivos por varios meses durante el tiempo más agudo de la pandemia en Colombia, la mayoría de las personas participantes de la encuesta percibieron que la salud vascular permaneció igual o mejoró. Pocos pacientes de este estudio tuvieron cancelación de los procedimientos y aunque la mayoría de estas las percibieron como no oportunas, hay que tener en cuenta que la atención ambulatoria estuvo cancelada y que se requirió un tiempo inicial de adaptación a la nueva situación por todos los actores del sistema de salud, tanto administrativo como asistencial y sobre todo los pacientes.

El uso de la telemedicina en la asistencia de pacientes durante la pandemia ha sido de vital importancia, y aunque ya se venía usando desde hace mucho tiempo, el impulso que debió dársele durante los últimos meses ha sido importante a nivel global, no solo para pacientes COVID-19 que pueden ser atendidos en su domicilio, sino en las otras patologías. Con esto se disminuye el contacto y la probabilidad de exposición y diseminación de la enfermedad, así como el uso de equipos personales de protección, optimiza los recursos locativos y de personal, todo esto en mejora de la calidad de la salud en estos tiempos difíciles ${ }^{8}$. Sin embargo, también quedan expuestos varios problemas en su implementación, como el bajo acceso a la tecnología necesaria o a la educación para su manipulación en zonas de bajos recursos o en personas con bajo nivel sociocultural principalmente, así como la regulación insuficiente de esta práctica o falta de estándares para que pueda ser aprovechada. Aunque no fue el objetivo principal de este estudio, se detectaron pocas consultas virtuales en los pacientes encuestados, pero es notorio resaltar que las capacidades en tecnología y recursos para realizar la atención virtual aumentaban a medida que mejoraba el grado de escolaridad y que, en general, los pacientes estuvieron satisfechos con su atención, pudiéndose resolver su requerimiento en más de la mitad de los pacientes. Esto puede deberse a una adaptación del pensamiento general respecto a las consultas virtuales por las condiciones globales durante la pandemia. Algunos estudios sobre la percepción de la atención por teleconsulta en cirugía vascular revelan que los pacientes percibieron la telemedicina útil y en ocasiones una mejor herramienta para lograr resolver sus requerimientos en salud, encontrando además una motivación adicional para la telemedicina la reducción en costos por los desplazamientos y la atención con menos tiempos de espera ${ }^{9,10}$.

Aunque no se precisó en este trabajo el método utilizado en la teleconsulta, hay reportes que muestran 
que las imágenes por celular pueden ser efectivas para seguimiento de úlceras, heridas postoperatorias y en algunos casos con apoyos diagnósticos, en enfermedades venosas y arteriales ${ }^{11,12}$.

Sin embargo, aunque entre los pacientes que fueron encuestados y que tuvieron evaluación virtual la satisfacción fue adecuada, es importante resaltar que un $80 \%$ no tuvo este tipo de atención, y del total el $20 \%$ no tenían acceso a la tecnología necesaria, por lo cual pudiera hacer falta delinear métodos uniformes para la realización de las consultas virtuales en cirugía vascular, definir cuáles pacientes pueden beneficiarse y aprovechar esta herramienta y cuáles no, ya que en los pacientes con edad avanzada y poco nivel socioeconómico o cultural, o que vivan en zonas apartadas, se puede dificultar la teleconsulta y que sea necesario implementar otras estrategias para la atención de estos pacientes.

Las patologías y consultas no emergentes por causas vasculares se vieron disminuidas y aplazadas en la etapa temprana de la pandemia ${ }^{13,14}$, al igual que lo encontrado en la población de esta serie, a los pacientes de todo el mundo se les agendaron citas virtuales durante la pandemia con el fin de evitar el contacto estrecho y reducir así la posibilidad de contagio por COVID-19 ${ }^{14}$.

Durante la pandemia por COVID-19 se han fortalecido los procedimientos endovasculares, ya que tienen un menor número de complicaciones e implican seguimiento ambulatorio, buscando minimizar la estancia hospitalaria y reducir la demanda en los servicios de cuidados intensivos ${ }^{15}$. Todo esto ha traído consigo implicaciones importantes a corto y largo plazo en cuestión de costos, por la reducción de procedimientos médicos y el menor tiempo de funcionamiento de los quirófanos. Un estudio evaluó la proyección de los procedimientos cancelados o pospuestos durante el pico más alto de la pandemia de 12 semanas en varios países, encontrando que pudiera ser de 28,404,603 procedimientos, la mayoría por enfermedad benigna. Con este dato ellos estiman que si los países aumentaran su capacidad quirúrgica en un $20 \%$ luego de la pandemia, les tomaría un promedio de 45 semanas ponerse al día con estos procedimientos ${ }^{16}$.

\section{Conclusión}

A pesar de la demora en la atención, el aumento de los síntomas en algunos pacientes y la reprogramación inoportuna de la consulta y los procedimientos, no se encontró un deterioro o complicaciones importantes en la salud vascular de los pacientes evaluados según su percepción. La adopción de consulta virtual pudo ayudar en la mayoría de los pacientes a resolver los requerimientos no urgentes. Sin embargo, es necesario mejorar los canales de comunicación virtuales, con el fin de proporcionar tranquilidad y una evaluación adecuada que permita identificar los pacientes con verdadero riesgo en los que la atención no pueda ser pospuesta. Ante la pregunta de cuál sería el impacto real en el pronóstico de los pacientes electivos en los que se ha diferido la atención por la pandemia y el costo que esto traería, aún falta más evidencia, y tal vez se requiera más tiempo para evaluar las consecuencias.

\section{Financiamiento}

La presente investigación no ha recibido ninguna beca específica de agencias de los sectores públicos, comercial o con ánimo de lucro.

\section{Conflicto de intereses}

Los autores declaran no tener conflicto de intereses.

\section{Responsabilidades éticas}

Protección de personas y animales. Los autores declaran que para esta investigación no se han realizado experimentos en seres humanos ni en animales.

Confidencialidad de los datos. Los autores declaran que han seguido los protocolos de su centro de trabajo sobre la publicación de datos de pacientes.

Derecho a la privacidad y consentimiento informado. Los autores han obtenido el consentimiento informado de los pacientes y/o sujetos referidos en el artículo. Este documento obra en poder del autor de correspondencia.

\section{Bibliografía}

1. Garza-Herrera R. El papel del especialista en angiología y cirugía vascular durante la pandemia por COVID-19. Rev Mex Angiol. 2020;48(1):38-40.

2. Correia M, Constâncio V, Silva JC, Lima P, Moreira M, Antunes LF, et al. Is there an impact of COVID-19 on admission of patients to the emergency department for vascular surgery? Ann Vasc Surg. 2020;69(August):100-4.

3. Utku UE, Levent MH, Zafer IH. Vascular surgery in the COVID-19 pandemic. J Vasc Surg. 2020;72(2):752-4.

4. Mavioğlu HL, Ünal EU, Așkın G, Küçüker --A, Özatik MA. Perioperative planning for cardiovascular operations in the COVID-19 pandemic. Turk Gogus Kalp Damar Cerrahisi Derg. 2020;28(2):236-43.

5. Bashar AHM, Hakim ME, Rahman MM, Dey NK, Chowdhury SMMH, Islam MM, et al. Vascular surgery practice guidelines during $\mathrm{CO}$ VID-19 pandemic in a setting of high work volume against limited resources: Perspective of a developing country. Ann Vasc Surg. 2021;70:306-13. 
Rev Mex Angiol. 2021;49(4)

6. American College of Surgeons. COVID-19 Guidelines for Triage of Vascular Surgery Patients. 2020 [Internet]. American College of Surgeons [consultado: 2 abril 2020]. Disponible en: https://www.facs.org/covid-19/ clinical-guidance/elective-case/vascular-surgery

7. Mouawad NJ, Woo K, Malgor RD, Wohlauer MV, Johnson AP, Cuff RF, et al. The impact of the COVID-19 pandemic on vascular surgery practice in the United States. J Vasc Surg. 2021;73(3):772-9.e4.

8. Ena J. Telemedicina aplicada al covid. Rev Clin Esp. 2020;220(8):501-2

9. Mishra V. Factores affecting the adoption of telemedicine during COVID-19. Indian J Public Health. 2020;64(Supp):S234-S236.

10. Li HL, Chan Y, Huang J, Cheng S. Pilot study using telemedicine video consultation for vascular patient's care during the COVID-19 period. Ann Vasc Surg. 2020;68:76-82.

11. Wiseman JT, Fernandes-Taylor S, Gunte R, Barnes M, Saunders R, Rathouz P. Inter-rater agreement and checklist validation for postoperative wound assessment using smartphone images in vascular surgery. J Vasc Surg Venous Lymphat Disord. 2016;4(3):320-8.e2
12. Paquette $\mathrm{S}$, Lin JC. Outpatient telemedicine program in vascular surgery reduces patient travel time, cost, and environmental pollutant emissions. Ann Vasc Surg. 2019;59:167-72.

13. Bath J, Aziz F, Smeds MR. Progression of changes in vascular surgery practices during the novel corona virus SARS-CoV-2 pandemic. Ann Vasc Surg. 2021 Apr 6;S0890-5096(21)00232-6. doi: 10.1016/j. avsg.2021.03.002. Online ahead of print.

14. Ng JJ, Ho P, Dharmaraj RB, Wong JCL, Choong AMTL. The global impact of COVID-19 on vascular surgical services. J Vasc Surg 2020;71:2182-3 e1

15. Vascular and Endovascular Research Network (VERN) COVER study collaborative. Global impact of the first coronavirus disease 2019 (COVID-19) pandemic wave on vascular services. $\mathrm{Br} J$ Surg. 2020;107(11):1396-400.

16. COVIDSurg Collaborative. Elective surgery cancellations due to the COVID-19 pandemic: global predictive modelling to inform surgical recovey plans. Br J Surg. 2020;107(11):1440-9. 